\title{
A Calm, Dispassionate Look at Skin Microbiota in Atopic Dermatitis: An Integrative Literature Review
}

Pengjie Wan · Ji Chen

Received: November 20, 2019 / Published online: January 20, 2020

(C) The Author(s) 2020

\section{ABSTRACT}

Atopic dermatitis (AD) is a chronic common inflammatory skin disorder with clinical characteristics of pruritic, dry, and recurrent flares that involve the whole body. Recent studies have demonstrated that the skin microbiota, characterized by an overgrowth of Staphylococcus aureus (S. aureus), plays a critical role in the manifestation of AD. There is striking evidence that skin microbiota can modulate the development and progression of $\mathrm{AD}$. Therefore, more and more therapeutic approaches are adopted for modifying skin microbiota. Here we discuss the role of skin microbiota in the etiology and maintenance of $\mathrm{AD}$; furthermore, we summarize the effects of therapeutic treatments on skin microbiota in $\mathrm{AD}$ based on published literature. With the help of the theoretical guidance suggested by microbial metagenome analysis, the reconstitution of microbiota should be a promising way to harness the pathogens of $\mathrm{AD}$ and could be used as a brandnew therapeutic strategy in clinical trials. We believe that the targeted therapy of dysbiosis in

Enhanced Digital Features To view enhanced digital features for this article go to https://doi.org/10.6084/ m9.figshare.11494461.

P. Wan $\cdot$ J. Chen $(\bowtie)$

Department of Dermatology, Shanghai Children's

Medical Center, Shanghai Jiao Tong University

School of Medicine, Shanghai, China

e-mail: chenji@scmc.com.cn
AD may possibly become a unique approach to an integrated treatment program in the near future.

Keywords: Atopic dermatitis; Skin microbiome; Skin microbiota; Staphylococcus aureus; Therapy

\section{Key Summary Points}

Skin microbiota is a complex ecosystem composed of bacteria, fungi, and viruses. Integrity of the diverse microbes plays essential role in maintaining homeostasis and preventing pathogens from invading skin. Recently, researchers found that increased colonization of Staphylococcus aureus (S. aureus) plays a critical role in the pathogenesis of atopic dermatitis (AD).

In this review, we summarize the clinical features of imbalanced skin microbiota associated with $\mathrm{AD}$, and we emphasize the effects of each therapeutic treatment and their influences on skin microbiota. Besides traditional treatment approaches, such as emollient, antibacterial treatment, tacrolimus, narrowband UVB, coal tar, biological therapy, and contact with nature, we also discuss novel treatments that are targeting specific strains of human microbiota. Intriguingly, the targeted therapy of dysbiosis in AD has the potential to become an integrated treatment in the near future. 


\section{INTRODUCTION}

Skin microbiota is a complex ecosystem composed of bacteria, fungi, and viruses. Balance and integrity of the diverse microbes play an essential role in preventing pathogens from invading skin [1], while the skin microbiome refers to the composition of all microbial genes in the skin's community [2]. Atopic dermatitis (AD), a worldwide heterogeneous, recurrent, chronic pruritic disease due to epithelial barrier dysfunction, immune dysregulation, and skin inflammation with dysbiosis of skin microbiota and colonization by the predominant pathogen $S$. aureus, shows an increasing tendency $[3,4]$. Many studies have found alterations in the composition of the microbiome in patients with $\mathrm{AD}$ compared with that of healthy individuals, and microbiota differs between lesional skin and non-lesional skin $[5,6]$. AD can also affect the skin of any part of the body, but it generally shows age-related morphological and distributional characteristics [7]. With the development of new technology, the application of whole genome sequencing (WGS) allows access to all genes of virtually all inflammation with dysbiosis of skin microbiota. Next-generation sequencing (NGS) technology in clinical bacteriology has been interrogating thousands of $16 \mathrm{~S}$ ribosomal RNA gene amplicons from bacteria to archaea in floras from a patient with $\mathrm{AD}[8,9]$. Currently, exploration of the skin microbiome in $\mathrm{AD}$ is attracting more and more attention from researchers. Dysbiosis in microbiota has been universally considered an important factor in the pathogenesis of $\mathrm{AD}$, and how to achieve and maintain a balance between skin microbiota and the host has become a hot research topic in the field of $\mathrm{AD}$ treatment [10]. Although traditional treatments including topical glucocorticoid and calcineurin inhibitors can inhibit inflammation, the recurrence of $\mathrm{AD}$ continues. Focusing on microbiota therapy is currently being developed to revise skin dysbiosis related to $\mathrm{AD}$, with the expectation of obtaining long-term remission. In this review, we give a brief introduction on clinical features of $\mathrm{AD}$, then discuss the pathogenesis of skin microbiota in $\mathrm{AD}$, and finally discuss the treatment strategies based on the skin microbiome by reviewing literature published till October 2019. This article is based on previously conducted studies and does not contain any studies with human participants or animals performed by any of the authors.

\section{CLINICAL FEATURES OF AD AND SKIN MICROBIOME}

The consistent increment in the prevalence of AD in both developing and developed countries has depicted a global trend in the burden of $\mathrm{AD}$, and the picture in the developing world may soon resemble that in developed countries [11], where $15-20 \%$ of children have been affected $[12,13]$. Nearly $70 \%$ of children with AD show disappearance of symptoms or spontaneous remission during puberty even in individuals with genetic heterogeneity of filaggrin mutations [14]. However, about $2-5 \%$ of adults worldwide can be affected and present as adultonset $\mathrm{AD}$ or infantile/childhood $\mathrm{AD}$, and the dermatitis symptoms may persist or recur over many years $[15,16]$.

Newborns generally carry an adequately uniform microbiome. The microbial community composition of newborns is initially determined by the manner of delivery, i.e., newborns delivered naturally obtain bacteria in their mother's vagina that are dominated by Lactobacillus, while those born by Caesarean section should inherit microbiota resembling those on their mothers' skin [17]. Although the skin lesions of infants are rather diffuse, it should be noted that most of the lesions occur on their faces and extensor sides of extremities [18]. Skin lesions on adults vary from a small localized plaque to a widespread lichenification, and even erythroderma. Shi et al. in 2016 identified that the skin microbiome of $\mathrm{AD}$ was significantly different between young children and adults-teenagers [14]. The skin microbiome during $\mathrm{AD}$ flares was investigated at species level by Byrd et al. [9]; dynamics of the microbial community from the pediatric $\mathrm{AD}$ group was consecutively monitored throughout the course of the disease. Eventually, they found a great dominance of $S$.aureus in those with more 
severe $\mathrm{AD}$; however, there was an obvious dominance of Staphylococcus epidermidis (S. epidermidis) in patients with mild symptoms.

\section{PATHOGENESIS OF AD REVEALED BY SKIN MICROBIOME}

\author{
Skin Microbiome Profiling in Patients \\ with AD
}

The pathophysiology of $\mathrm{AD}$ is associated with genetic, immunological, environmental, and microbiota risk factors. Aberrations of the immune system have long been considered as having key roles, until recently; molecular epidemiology has tended to focus on the skin microbiome as a common precursor and pathological feature. At present, the pathology of $\mathrm{AD}$ includes three critical factors: impaired skin barrier function, microbial dysbiosis, and cutaneous immune abnormality predisposed to T helper 2 (Th2) immunity, which may aggravate one another $[19,20]$.

The microbiota dysbiosis in $\mathrm{AD}$ is generally thought to be strongly related to the colonization of $S$. aureus on the skin, and the colonized skin lesions are closely correlated with the relative level of $S$.aureus $[21,22]$. S. aureus was abundant on the skin of patients with $\mathrm{AD}$ compared with normal controls [6, 14]. The affected skin sites, especially inflamed areas, were predominantly colonized by more $S$. aureus compared to unaffected sites in patients with AD $[9,14]$. The abundance of Staphylococcus and Corynebacterium spp. increased dramatically in untreated patients during a flare, while bacterial diversity was decreased including the reduction of Propionibacterium, Streptococcus, Acinetobacter, and Corynebacterium spp. [4, 21]. Greater bacterial diversity with increased abundance of Staphylococcus, Streptococcus, Corynebacterium, and Propionibacterium spp. was observed after AD treatment with clinical remission, suggesting a positive correlation with disease severity [23]. It is reported in a metaanalysis of 95 observational studies that the pooled $S$. aureus colonized $70 \%$ of lesional skin, $39 \%$ of nonlesional skin, $62 \%$ of nasal colonized patients with $\mathrm{AD}$, and $3 \%$ of nonatopic patients [6].

Kobayashi et al. in 2015 [24] demonstrated that natural occurrence of S. aureus and colonization of Corynebacterium bovis caused inflammation of eczematous dermatitis in a mice model that resembled that observed in patients with AD. Thus, the dysbiosis is regarded as a pathological factor that can drive eczematous inflammation in mice.

\section{S. aureus}

Staphylococcus aureus is a well-known grampositive spherical bacterium that occurs in grape-like clusters when viewed under a microscope. It is a facultative aerobic coccus with positive coagulase reaction. This bacterium is a clinically unapparent "carrier" and could lead to infection and thus it has been depicted as a pathobiont.

The severity of $\mathrm{AD}$ is usually associated with biofilms formed by $S$. aureus [25]. A biofilm is a bacterial assemblage attached to the surface and enclosed in an extracellular matrix. Research has shown that biofilms induce immune evasion, leading to the recurrence of $\mathrm{AD}$ and hardto-treat infections [26].

All S. aureus strains express superantigens including staphylococcal enterotoxin (SE) and toxic shock syndrome toxin-1 (TSST-1). The colonization of $S$. aureus could exacerbate $\mathrm{AD}$ and cause vicious cycles via inducing thymic stromal lymphopoietin (TSLP) and Th2/Th17type inflammation [9].

Recently, it has been proven that S.aureus plays a crucial role in occurrence and development of Th2 skewing and skin inflammation by multiple ways including the induction of $\mathrm{T}$ cellindependent B cell expansion, the release of cytokine and proinflammatory lipoproteins, and furthermore the stimulation of mast cell degranulation [23, 27], leading to the release of characteristic interleukins (ILs), such as IL-31, a notable pruritus-related cytokine [27, 28] (Fig. 1). When $S$. aureus from patients with AD was transplanted to a mice model, the epidermides of mice were thickened and cutaneous Th2 and Th17 cells emerged and expanded [23]. 
The allergens, especially superantigens, can initiate immunoglobulin E (IgE) responses to histamine derived from the mast cells and basophils. The production of S. aureus superantigen definitely facilitates the maturation of Th2 cells, and eventually leads to inflammatory response. Moreover, the colonization of S. aureus on skin can produce multiple virulence factors such as toxins and enzymes contributing to the inflammation and skin barrier dysfunction, and consequently cause bacteremia and sepsis through the invasion of human skin infection by $S$. aureus.

\section{S. epidermidis}

Staphylococcus epidermidis is considered a skin commensal, but its role in AD is unclear. Besides $S$. aureus, it is increased from the reports on colonization of $S$. epidermidis on lesional skin of pediatric AD. Interestingly, S. epidermidis skin colonization in $\mathrm{AD}$ can produce antimicrobial peptides (AMP) that could selectively target $S$. aureus [29]. It implies a compensatory mechanism of $S$. epidermidis in controlling pathogens.

\section{TREATMENT TARGETING SKIN MICROBIOTA}

To extend a patient's symptom-free period for as long as possible, multifactorial approaches are essential for the treatment of $\mathrm{AD}$ disease because of its multifactorial nature. The rationale of targeting the microbiota in $\mathrm{AD}$ derives from considerable benefits that related to the microflora diversity [28]. The abundance of $S$. aureus is tightly associated with disease flares

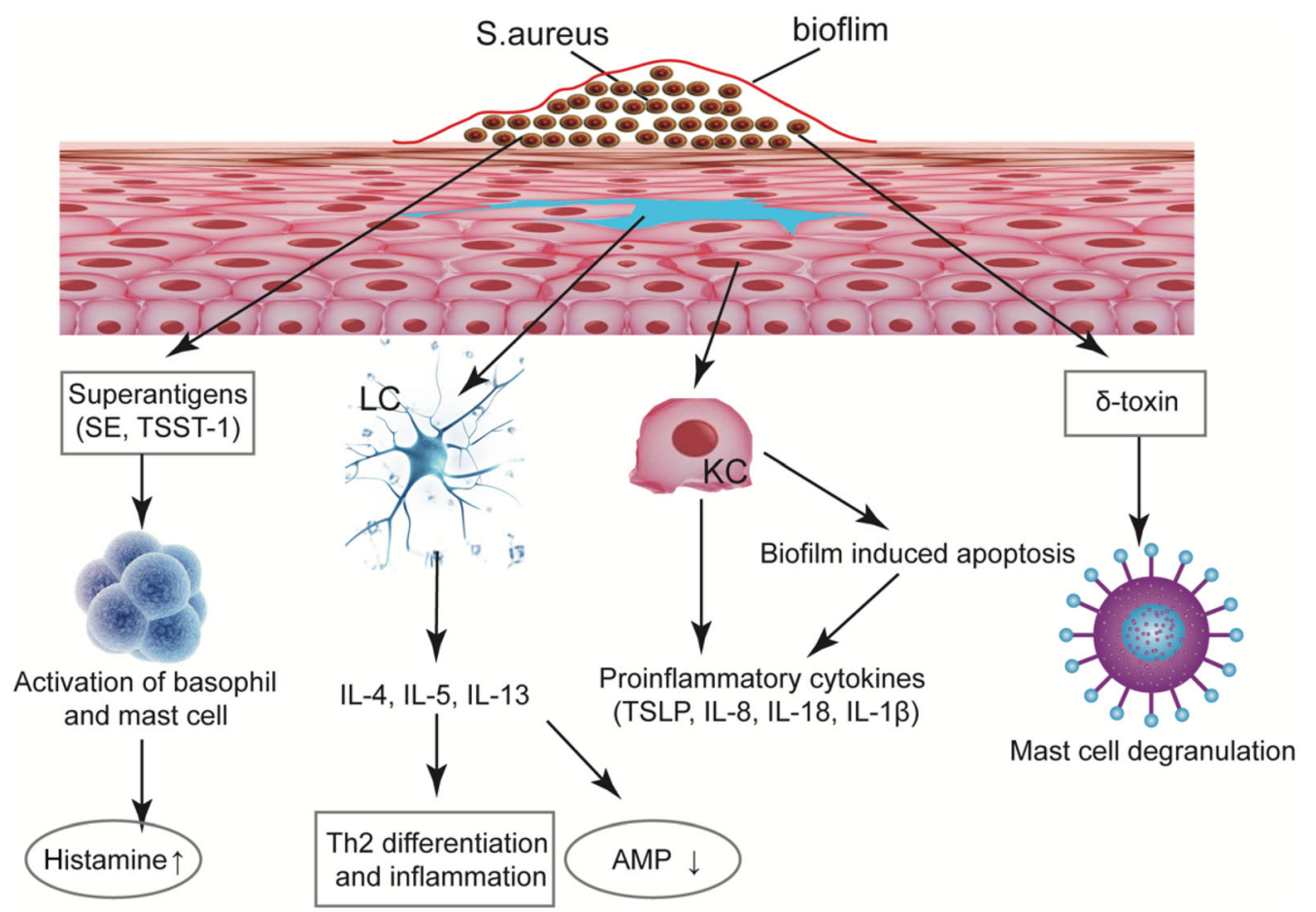

Fig. 1 Pathogenic role of Staphylococcus aureus involved in atopic dermatitis. $S$. aureus expresses superantigens, such as SE and TSST-1, which activate basophils and mast cell and cause histamine release; $S$. aureus activates LC releasing IL4, IL-5, and IL-13, which induces AMP reduction and Th2 differentiation and inflammation; $S$. aureus stimulates $\mathrm{KC}$ releasing proinflammatory cytokines including TSLP,
IL-8, IL-18, and IL-1 $\beta$ and biofilm can induce KC apoptosis releasing inflammatory cytokines; $S$. aureus can release $\delta$-toxin inducing mast cell degranulation. SE staphylococcal enterotoxin, TSST-1 toxic shock syndrome toxin-1, LC Langerhans cell, TSLP thymic stromal lymphopoietin, IL interleukin, KC keratinocyte 
and therapeutic responses [30]. Microbial diversity during $\mathrm{AD}$ flares is strongly correlated with recent $\mathrm{AD}$ treatments. It is likely that treatment induces changes in skin bacterial diversity. It indicates that $\mathrm{AD}$ treatments diversify skin bacteria preceding clinical improvements in disease activity [21]. Here we describe all kinds of strategies used for treatment targeting skin microbiota in patients with $\mathrm{AD}$.

\section{Emollient Use}

Considering that barrier dysfunction with epidermal water loss has an important relationship with $\mathrm{AD}$, topical treatment using emollient is usually encouraged as a basic therapy $[3,31,32]$. Glatz et al. in 2018 conducted an experiment in 6-month-old infants with family history of $\mathrm{AD}$ [33]. The emollient group demonstrated higher bacterial diversity, decreased skin $\mathrm{pH}$, and increased proportion of S. salivarius. It was a remarkable benefit of applying emollients in high-risk infants. Further studies are needed to confirm the preliminary results of emollients as a prophylactic therapy for AD [33]. Seite et al. in 2014 reported that when treated with emollients, the clinical symptoms of $\mathrm{AD}$ were alleviated in $72 \%$ of patients with $\mathrm{AD}$, as indicated by an increased overall diversity and a decrease in the abundance of Staphylococcus species. Importantly, the microbial communities on the affected skin in the treatment group more closely resembled those on unaffected skin [5].

\section{Antibacterial Treatment}

It is essential to apply antibacterial agents such as antiseptics and antibiotics in clinically relevant bacterial infection in $\mathrm{AD}$, and in patients with signs of bacterial superinfections, intermittent bleach baths and intranasal application of mupirocin ointment decreased the severity of AD [34]. However, it should be noted that the use of antibiotics in the treatment of systematic decontaminations of $S$. aureus is controversial as large-scale use of antibiotics would result in bacterial resistance and microbiota disorder [35].
Although bleach baths are widely accepted as an effective therapy for $\mathrm{AD}$ in some areas [36], they still remain highly controversial worldwide. Moreover, bleach baths were not more effective than water baths alone in reducing the severity of $\mathrm{AD}$ [37]. Moreover, sometimes the effects of bleach bath were contradictory in human studies. Gonzalez et al. in 2016 found that a 4-week duration period and/or twiceweekly baths may not be sufficient for additional impact on the cutaneous microbiome [38]. Another study evaluated the bleach bath by reviewing the effect of either topical corticosteroid alone or in combination with dilute bleach baths. The results showed improved clinical eczema representation and suppressed Staphylococcus species on lesional and non-lesional sites in both groups, suggesting an invalid effect of the additional dilute bleach baths [23]. Some researchers even expressed a word of caution based on the potential dangers in the application of such a strongly alkaline treatment [39].

\section{Tacrolimus Ointment for Treatment of AD}

Tacrolimus ointment, which is a very important anti-inflammatory topical drug, is recommended as the first-line treatment for $A D$ with topical corticosteroids [40]. Wongpiyabovorn et al. [41] conducted an experiment with nine Thai patients with $\mathrm{AD}$ undergoing a 4-week monotherapy with tacrolimus. A lower prevalence of the phyla Actinobacteria and Proteobacteria and a higher prevalence of the phylum Firmicutes were identified in patients with AD. With the notable improvement of all clinical scores, there was a significant increase in some commensal genera including Dermacoccus, Pseudomonas, Corynebacterium, Proteus, Micrococcus luteus, and Lactococcus in the patients with $\mathrm{AD}$ after treatments with tacrolimus ointment, which should reflect the effect of tacrolimus on the skin microbiome. This study provides unequivocal proof that tacrolimus has a positive impact on the skin microbiome in patients with AD. 


\section{Narrowband Ultraviolet B (UVB) Phototherapy}

UVB phototherapy is well established as a second-line treatment for adolescent and adult patients with AD. Kwon et al. carried out research on 18 patients with $\mathrm{AD}$ by using UVB phototherapy. A drastic increase in microbial diversity and decrease of $S$. aureus proportion were observed with clinical improvement of AD. The UVB treatment did not exert additive effects on the improvement of $\mathrm{AD}$; however, it seemed to reduce $\mathrm{AD}$ recurrence [42].

\section{Treatment with Coal Tar}

Coal tar (CT), which is an ancient yet highly effective and safe therapeutic agent, was applied to seven patients with $\mathrm{AD}$ and ten healthy volunteers with vehicle treatment as control [22]. During CT treatment, the abundance of Staphylococcus decreased, whereas Propionibacterium abundance increased. The therapeutic effect of CT treatment was achieved by targeting the cutaneous bacterial composition through keratinocyte-derived AMP production via activation of the aryl hydrocarbon receptor (AHR).

\section{Biological Therapy in Patients with AD}

Dupilumab, a monoclonal antibody against interleukin-4 receptor alpha, has been used in numerous allergic diseases and works by blocking signaling of interleukin-4 and interleukin13 , type $2 / \mathrm{Th} 2$ cytokines. It has been confirmed to be effective in patients with moderate to severe $\mathrm{AD}$ in several double-blind, placebocontrolled studies and in clinical practice [43-45]. The result showed that clinical improvement is positively correlated with increased favorable microbial community and reduced abundance of $S$. aureus [46]. More novel systemic immunomodulatory agents for $\mathrm{AD}$ are under development, and it is promising that these therapies will not only improve skin immunity but also adjust $S$. aureus colonization by modulating their cell wall proteins [19].

\section{Contact with Nature}

A new theory is that regular contact with nature could enhance the diversity of microbiota [47]. For example, dog ownership confers a protective effect against $\mathrm{AD}$ development because dog owners spend more time outdoors and therefore have more exposure to natural environments, resulting in the alteration of their skin microbiota. Moreover, pet dogs also benefit owners' health by reducing psychological stress and promoting associated immunomodulatory effects $[48,49]$.

\section{Novel Treatments}

Interestingly enough, novel treatment strategies seek to target specific aspects of dysbiosis in $\mathrm{AD}$ [29]. The commensal strains of coagulase-negative staphylococci (CoNS), collected from the skin of healthy individuals, protected against skin dysbiosis by reducing the colonization of S. aureus in patients with $\mathrm{AD}$ [29]. A prospective, double-blind, placebo-controlled clinical study in 75 volunteers with $\mathrm{AD}$ showed that cream containing $5 \%$ of the lysate of a nonpathogenic proteobacterium Vitreoscilla filiformis could significantly improve a clinical score and reduce transepidermal water loss (TEWL), itch, and insomnia compared with placebo [50]. A further study showed that topical transplantation with Roseomonas mucosa was effective in decreasing disease severity, topical steroid requirement, and $S$. aureus burden in patients with AD. There were no adverse effects or treatment complications in this study [51]. Overall, more research is to enable us to implement such skin microbiota as a therapeutic target in the pathogenesis and treatment of $\mathrm{AD}[30]$.

\section{CONCLUSIONS}

New insights into the pathophysiology of AD focus on the important role of microbial dysbiosis, which was a driving factor in the pathogenesis of $\mathrm{AD}$ according to animal experiments [4]. However, it is still unclear if causal relationships between the skin microbiota and 
disease exist in patients with AD. S. aureus plays a critical role not only during $\mathrm{AD}$ flare but also in the treatment of skin dysbiosis. Treatment targeting the microbiota in patients with $\mathrm{AD}$ can effectively reduce the colonization of $S$. aureus and significantly increase the diversity of bacteria on the skin surface, suggesting an important role of microbial dysbiosis in the pathogenesis of AD. Thus, the establishment of the balance of skin microbiota in patients with $\mathrm{AD}$ is feasible for the treatment and prevention of AD disease. Pan-genome and comparative genome analyses of the specific bacterial species should reveal their genomic abundance in the healthy and diseased human skin microbiota, and all the skin microbiome of AD disease must be investigated in the context of the healthy skin microbiota. Probing the molecular mechanism of interaction between $S$. aureus from AD skin and the commensal strains will be a major focus for the future.

\section{ACKNOWLEDGMENTS}

We thank Chaoyong Li for the help with mapping; we thank Dr. Yanxia Wei and Dr. Ruifang Liu for the revision of the manuscript.

Funding. This study the journal's Rapid Service Fee were supported by Foundation of Shanghai Children's Medical Center, School of Medicine, Shanghai Jiaotong University (No. YJSCMC2017-12). No funding or sponsorship was received for this study.

Authorship. All named authors meet the International Committee of Medical Journal Editors (ICMJE) criteria for authorship for this article, take responsibility for the integrity of the work as a whole, and have given their approval for this version to be published.

Disclosures. Pengjie Wan and Ji Chen have nothing to disclose.

Compliance with Ethics Guidelines. This article is based on previously conducted studies and does not contain any studies with human participants or animals performed by any of the authors.

Data Availability. The datasets generated during and analyzed during the current study are available from the corresponding author on reasonable request.

Open Access. This article is licensed under a Creative Commons Attribution-NonCommercial 4.0 International License, which permits any non-commercial use, sharing, adaptation, distribution and reproduction in any medium or format, as long as you give appropriate credit to the original author(s) and the source, provide a link to the Creative Commons licence, and indicate if changes were made. The images or other third party material in this article are included in the article's Creative Commons licence, unless indicated otherwise in a credit line to the material. If material is not included in the article's Creative Commons licence and your intended use is not permitted by statutory regulation or exceeds the permitted use, you will need to obtain permission directly from the copyright holder. To view a copy of this licence, visit http://creativecommons.org/licenses/by$\mathrm{nc} / 4.0 /$.

\section{REFERENCES}

1. Chen YE, Fischbach MA, Belkaid Y. Skin microbiota-host interactions. Nature. 2018;553:427-36.

2. Byrd AL, Belkaid Y, Segre JA. The human skin microbiome. Nat Rev Microbiol. 2018;16:143-55.

3. Sala-Cunill A, Lazaro M, Herraez L, et al. Basic skin care and topical therapies for atopic dermatitis: essential approaches and beyond. J Investig Allergol Clin Immunol. 2018;28:379-91.

4. Bjerre RD, Bandier J, Skov L, Engstrand L, Johansen JD. The role of the skin microbiome in atopic dermatitis: a systematic review. Br J Dermatol. 2017;177:1272-8.

5. Seite S, Flores GE, Henley JB, et al. Microbiome of affected and unaffected skin of patients with atopic dermatitis before and after emollient treatment. J Drugs Dermatol. 2014;13:1365-72. 
6. Totte JEE, van der Feltz WT, Hennekam M, van Belkum A, van Zuuren EJ, Pasmans S. Prevalence and odds of Staphylococcus aureus carriage in atopic dermatitis: a systematic review and meta-analysis. Br J Dermatol. 2016;175:687-95.

7. Weidinger $S$, Novak N. Atopic dermatitis. Lancet. 2016;12:1109-22.

8. Rapin A, Pattaroni C, Marsland BJ, Harris NL. Microbiota analysis using an Illumina MiSeq platform to sequence $16 \mathrm{~S}$ rRNA genes. Curr Protoc Mouse Biol. 2017;7:100-29.

9. Byrd AL, Deming C, Cassidy SKB, et al. Staphylococcus aureus and Staphylococcus epidermidis strain diversity underlying pediatric atopic dermatitis. Sci Transl Med. 2017;9:eaal4651.

10. Fyhrquist N, Muirhead G, Prast-Nielsen S, et al. Microbe-host interplay in atopic dermatitis and psoriasis. Nat Commun. 2019;10:4703.

11. Flohr C, Mann J. New insights into the epidemiology of childhood atopic dermatitis. Allergy. 2014;69:3-16.

12. Tsakok T, Woolf R, Smith $\mathrm{CH}$, Weidinger S, Flohr C. Atopic dermatitis: the skin barrier and beyond. Br J Dermatol. 2019;180:464-74.

13. Garg N, Silverberg JI. Epidemiology of childhood atopic dermatitis. Clin Dermatol. 2015;33:281-8.

14. Shi B, Bangayan NJ, Curd E, et al. The skin microbiome is different in pediatric versus adult atopic dermatitis. J Allergy Clin Immunol. 2016;138: 1233-6.

15. Katsarou A, Armenaka M. Atopic dermatitis in older patients: particular points. J Eur Acad Dermatol Venereol. 2011;25:12-8.

16. Wollenberg A, Oranje A, Deleuran M, et al. ETFAD/ EADV Eczema task force 2015 position paper on diagnosis and treatment of atopic dermatitis in adult and paediatric patients. J Eur Acad Dermatol Venereol. 2016;30:729-47.

17. Powers CE, McShane DB, Gilligan PH, Burkhart CN, Morrell DS. Microbiome and pediatric atopic dermatitis. J Dermatol. 2015;42:1137-42.

18. Lyons JJ, Milner JD, Stone KD. Atopic dermatitis in children: clinical features, pathophysiology, and treatment. Immunol Allergy Clin North Am. 2015;35:161-83.

19. Iwamoto K, Moriwaki M, Miyake R, Hide M. Staphylococcus aureus in atopic dermatitis: strainspecific cell wall proteins and skin immunity. Allergol Int. 2019;68:309-15.
20. Akiyama M. Corneocyte lipid envelope (CLE), the key structure for skin barrier function and ichthyosis pathogenesis. J Dermatol Sci. 2017;88:3-9.

21. Kong HH, Oh J, Deming C, et al. Temporal shifts in the skin microbiome associated with disease flares and treatment in children with atopic dermatitis. Genome Res. 2012;22:850-9.

22. Smits JPH, Ederveen THA, Rikken G, et al. Targeting the cutaneous microbiota in atopic dermatitis by coal tar via AHR-dependent induction of antimicrobial peptides. J Invest Dermatol. 2019. https:// doi.org/10.1016/j.jid.2019.06.142.

23. Kim J, Kim BE, Leung DYM. Pathophysiology of atopic dermatitis: clinical implications. Allergy Asthma Proc. 2019;40:84-92.

24. Kobayashi T, Glatz M, Horiuchi K, et al. Dysbiosis and Staphylococcus aureus colonization drives inflammation in atopic dermatitis. Immunity. 2015;42:756-66.

25. Kim J, Kim H. Microbiome of the skin and gut in atopic dermatitis (AD): understanding the pathophysiology and finding novel management strategies. J Clin Med. 2019;8:444.

26. Tankersley A, Frank MB, Bebak M, Brennan R. Early effects of Staphylococcus aureus biofilm secreted products on inflammatory responses of human epithelial keratinocytes. J Inflamm (Lond). 2014;11: 17.

27. Geoghegan JA, Irvine AD, Foster TJ. Staphylococcus aureus and atopic dermatitis: a complex and evolving relationship. Trends Microbiol. 2018;26: 484-97.

28. Stalder JF, Fluhr JW, Foster T, Glatz M, Proksch E. The emerging role of skin microbiome in atopic dermatitis and its clinical implication. J Dermatol Treat. 2019;30:357-64.

29. Nakatsuji T, Chen TH, Narala S, et al. Antimicrobials from human skin commensal bacteria protect against Staphylococcus aureus and are deficient in atopic dermatitis. Sci Transl Med. 2017;9(378): eaah4680.

30. Pothmann A, Illing T, Wiegand C, Hartmann AA, Elsner P. The microbiome and atopic dermatitis: a review. Am J Clin Dermatol. 2019;20:749-61.

31. Chylla R, Schnopp C, Volz T. Basic skin care in atopic dermatitis-new and established treatment options. J Dtsch Dermatol Ges. 2018;16:976-9.

32. Shaker M, Murray RGP, Mann JA. The ins and outs of an 'outside-in' view of allergies: atopic dermatitis 
and allergy prevention. Curr Opin Pediatr. 2018;30: 576-81.

33. Glatz M, Jo JH, Kennedy EA, et al. Emollient use alters skin barrier and microbes in infants at risk for developing atopic dermatitis. PLoS One. 2018;13: e0192443.

34. Huang JT, Abrams M, Tlougan B, Rademaker A, Paller AS. Treatment of Staphylococcus aureus colonization in atopic dermatitis decreases disease severity. Pediatrics. 2009;123:e808-14.

35. Darsow U, Wollenberg A, Simon D, et al. ETFAD/ EADV eczema task force 2009 position paper on diagnosis and treatment of atopic dermatitis. J Eur Acad Dermatol Venereol. 2010;24:317-28.

36. Ryan C, Shaw RE, Cockerell CJ, Hand S, Ghali FE. Novel sodium hypochlorite cleanser shows clinical response and excellent acceptability in the treatment of atopic dermatitis. Pediatr Dermatol. 2013;30:308-15.

37. Chopra R, Vakharia PP, Sacotte R, Silverberg JI. Efficacy of bleach baths in reducing severity of atopic dermatitis: a systematic review and metaanalysis. Ann Allergy Asthma Immunol. 2017;119: 435-40.

38. Gonzalez ME, Schaffer JV, Orlow SJ, et al. Cutaneous microbiome effects of fluticasone propionate cream and adjunctive bleach baths in childhood atopic dermatitis. J Am Acad Dermatol. 2016;75(481-93):e8.

39. Barnes TM, Greive KA. Use of bleach baths for the treatment of infected atopic eczema. Australas J Dermatol. 2013;54:251-8.

40. Ohtsuki M, Morimoto H, Nakagawa H. Tacrolimus ointment for the treatment of adult and pediatric atopic dermatitis: review on safety and benefits. J Dermatol. 2018;45:936-42.

41. Wongpiyabovorn J, Soonthornchai W, Wilantho A, et al. Effect of tacrolimus on skin microbiome in atopic dermatitis. Allergy. 2019;74:1400-6.

42. Kwon S, Choi J, Shin J, et al. Changes in lesional and non-lesional skin microbiome during treatment of atopic dermatitis. Acta Derm Venereol. 2019;99:284-90.

43. Fargnoli MC, Esposito M, Ferrucci S, et al. Real-life experience on effectiveness and safety of dupilumab in adult patients with moderate-to-severe atopic dermatitis. J Dermatol Treat. 2019. https:// doi.org/10.1080/09546634.2019.1682503.

44. Cork MJ, Thaci D, Eichenfield LF, et al. Dupilumab in adolescents with uncontrolled moderate-to-severe atopic dermatitis: results from a phase IIa openlabel trial and subsequent phase III open-label extension. Br J Dermatol. 2020;182(1):85-96.

45. Ariens LFM, van der Schaft J, Bakker DS, et al. Dupilumab is very effective in a large cohort of difficult-to-treat adult atopic dermatitis patients: first clinical and biomarker results from the BioDay registry. Allergy. 2019. https://doi.org/10.1111/all. 14080.

46. Callewaert C, Nakatsuji T, Knight R, et al. IL-4Ralpha blockade by dupilumab decreases Staphylococcus aureus colonization and increases microbial diversity in atopic dermatitis. J Invest Dermatol. 2020;140(1):191-202.e7.

47. Stefanovic N, Flohr C, Irvine AD. The exposome in atopic dermatitis. Allergy. 2019. https://doi.org/10. 1111/all.13946.

48. Nisbet EK, Zelenski JM, Murphy SA. The nature relatedness scale linking individuals' connection with nature to environmental concern and behavior. Environ Behav. 2009;41:715-40.

49. Langan SM, Flohr C, Williams HC. The role of furry pets in eczema: a systematic review. Arch Dermatol. 2007;143:1570-7.

50. Gueniche A, Knaudt B, Schuck E, et al. Effects of nonpathogenic gram-negative bacterium Vitreoscilla filiformis lysate on atopic dermatitis: a prospective, randomized, double-blind, placebocontrolled clinical study. Br J Dermatol. 2008;159: 1357-63.

51. Myles IA, Earland NJ, Anderson ED, et al. First-inhuman topical microbiome transplantation with Roseomonas mucosa for atopic dermatitis. JCI Insight. 2018;3:e120608. 